\title{
Matrix Metalloproteinase-9 and Topical Cyclosporine Are Associated with Conjunctival Microbiota Culture Positivity in Korean Patients with Stevens-Johnson Syndrome
}

\section{Jayoon Moon}

Laboratory of Ocular Regenerative Medicine and Immunology, Seoul Artificial Eye Center, Seoul National University Hospital Biomedical Research Institute, Seoul

\section{Yunjin Lee}

Laboratory of Ocular Regenerative Medicine and Immunology, Seoul Artificial Eye Center, Seoul National University Hospital Biomedical Research Institute, Seoul

\section{Mee Kum Kim ( $\square$ kmk9@snu.ac.kr)}

Department of Ophthalmology, Seoul National University College of Medicine, Seoul

\section{Research Article}

Keywords: Conjunctiva, Cyclosporine, Matrix metalloproteinase-9, Microbiota, Stevens-Johnson syndrome

Posted Date: December 15th, 2021

DOI: https://doi.org/10.21203/rs.3.rs-1088309/v1

License: @ (i) This work is licensed under a Creative Commons Attribution 4.0 International License.

Read Full License 


\section{Abstract}

\section{Background}

Stevens-Johnson syndrome (SJS) is an abnormal immune-response causing extensive exfoliation of the mucocutaneous tissue including conjunctiva. While several factors are associated with the alteration of conjunctival microbiota, the conjunctiva of SJS patients are found to harbor a different microbiota compared to healthy subjects. We investigated the conjunctival microbiota of Korean SJS patients, and identified factors associated with the conjunctival microbiota and its positive culture.

\section{Methods}

Medical records were retrospectively reviewed in 30 SJS patients who had undergone conjunctival swab culture sampling. Chronic ocular surface complications score (COCS), tear break-up time (TBUT), tear matrix metalloproteinase 9 (MMP9), and results of conjunctival swab culture were assessed.

\section{Results}

Positive culture was seen in $58.1 \%$. Gram positive bacteria was most commonly isolated, among which Coagulase-negative Staphylococci (45.5\%) and Corynebacterium species (40.9\%) were predominantly observed. Tear MMP9 positivity was observed significantly more in the positive culture group (100\%) compared to the negative culture group $(75 \%)(P=0.040)$. In patients who had repetitive cultures, positive- persistence group had more patients using topical cyclosporine compared to the negativetransition group ( $90.0 \%$ vs $33.3 \%$, respectively, $P=0.041)$. No significant difference was found between COCS and conjunctival swab culture result, and the same as in TBUT and conjunctival swab culture result.

\section{Conclusion}

Our study suggest that tear MMP9 positivity may reflect the presence of an abnormal ocular surface microbiota and topical cyclosporine may be related to persistent culture positivity in SJS patients.

\section{Background}

Stevens-Johnson syndrome (SJS) is caused by an abnormal immune-response to drugs or other risk factors and is characterized with extensive exfoliation of the mucocutaneous tissue which can be fatal.[1, 2] Acute and chronic ocular manifestations may lead to chronic sequelae of limbal stem cell deficiency, corneal conjunctivalization, persistent epithelial defect, lid margin keratinization, symblepharon, ankyloblepharon etc.[3-5] Ocular involvement in SJS can be managed with surgical interventions and several medical therapies which includes topical corticosteroids or cyclosporine. $[4,6]$

An innumerable amount of microbial communities inhabit the human body including the ocular surface, especially the conjunctiva.[7] A normal conjunctiva exhibits diverse microorganisms that commonly 
consist bacteria such as Coagulase-negative Staphylococci, Staphylococcus group, Corynebacterium, Propionibacterium.[7-12] The conjunctival microbiota can be easily altered depending on factors such as use of contact lens, topical or systemic antibiotics, host's age, or presence of ocular surface diseases etc. $[8,9,13,14]$ Higher positive culture rate was observed in elder subjects or with diabetes.[15] Subjects with dry eye or blepharitis were reported to exhibit different conjunctival microbiota compared to healthy subjects.[14-17] In particular, the conjunctiva of SJS patients were found to harbor a significantly different microbiome and have higher culture-positive rate compared to healthy subjects. $[8,11,14,18]$

While the microbiome of several areas of the body have been studied to affect human diseases, the physiological role of ocular surface microbiome is yet unknown. Still, evidence show that an appropriate balance between the ocular surface microbiome and its mucosal immunity helps maintain the homeostasis of commensal bacteria and prevent opportunistic infections.[19] Likewise, a weakened and damaged ocular surface of SJS patients may be more prone to harbor pathobionts and allow opportunistic infections, which can also be aggravated by surgical interventions or medical therapy such as topical immunosuppressant or antibiotics.[19-23]

Herein, we investigated the conjunctival microbiota of Korean SJS patients using conventional swab cultures, and identified factors associated with the conjunctival culture results.

\section{Methods}

\section{Subjects and Study Design}

This study was approved by the Institutional Review Board of Seoul National University Hospital (IRB No. 2102-014-1193, Seoul, Republic of Korea) and was conducted with adherence to Declaration of Helsinki. The informed consent from patients was waived by the IRB because the study was based on the retrospective review of old charts.

This is a retrospective case-series study of SJS patients who had undergone conjunctival swab culture sampling between January 1st, 2019 and December 31st, 2020 at Seoul National University Hospital (Seoul, Republic of Korea). From medical chart review, the following data were collected: 1) general medical history and demographic information, 2) clinical characteristics from ocular examinations including COCS (range, $0-15$ ), TBUT and tear MMP9 elevation, and 3) conjunctival swab culture results. The eye with the highest COCS was chosen for analysis and if the scores were the same in both eyes the right eye was included. Excluded from analysis were patients under 18 years of age, with active infectious keratitis and with insufficient clinical data, such as conjunctival swab culture results and COCS.

COCS was evaluated according to previous studies in modifications based on the grading system by Sotozono et al.[24, 25] COCS, ranging from 0 to 15 , where 15 indicates the most severe ocular complication, was defined as the sum of the following components' scores: 1) conjunctival hyperemia (no $=0$, yes $=1), 2$ ) decreased tear volume (Schirmer strip test $\leq 1 \mathrm{~mm} / \mathrm{min}=1$ ), 3) eyelid involvement (trichiasis, distichiasis, or severe meibomian gland dysfunction: 1 for the presence of each component), 
4) corneal involvement (superficial punctate keratitis, corneal thinning, corneal opacity: 1 for the presence of each component), 5) limbal deficiency (partial corneal neovascularization $=1$, near total corneal neovascularization with persistent corneal epithelial defect $=2$, total conjunctivalization $=3$ ), and 6 ) symblepharon formation ( 1 for each quadrant involved, a total of 4 ).

TBUT was evaluated under slit lamp biomicroscopy with cobalt blue filter after application of fluorescein strip. TBUT was measured three consecutive times with a stop watch after each blink. The average of the three measurements was used for analysis.

MMP9 elevation to $\geq 40 \mathrm{ng} / \mathrm{ml}$ was tested using InflammaDry test (RPS Diagnostics; Sarasota, FL, USA) according to the manufacturer's instruction.[27, 28] The InflammaDry device was gently dabbed at multiple locations of the inferior tarsal conjunctiva with releasing the lid after every 2-3 dabs and allowing the patient to blink. After obtaining sufficient tear sample, the device was immediately loaded onto the test cassette and placed directly into the manufacturer's provided buffer solution. After 10 minutes, positivity for MMP9 $\geq 40 \mathrm{ng} / \mathrm{ml}$ was indicative when 1 blue line and 1 red line was present in the device's test result window.[27, 28]

\section{Conjunctival Swab Culture And Drug Sensitivity Test}

Conjunctival swab culture was performed initially before applying any eyedrops, including topical anesthesia or fluorescein strips. Conjunctival swab sampling from each eye from deep portions of the medial and lateral lower conjunctival fornix was carried out using a sterilized cotton tip for each site.[11] Careful caution was taken to avoid the sterilized cotton tip from being in possibly contaminated by factors, such as the eyelid skin or eyelash. After obtaining each swab sample, it was immediately inoculated directly onto either blood agar plate or Sabouraud's agar plate. When possible, a repetition of conjunctival swab culture was performed in the same manner at an interval of at least 3 months since last test.

Incubation for the growth of bacteria and fungus, and drug susceptibility tests were performed at the department of laboratory medicine at Seoul National University Hospital (Seoul, Republic of Korea). Blood agar plate was used to culture a wide range of bacteria, including fastidious microbes and those that are difficult to grow such as Streptococcus and Staphylococcus, and to differentiate hemolytic bacteria. Sabouraud's agar plate was used in cultivating fungus, such as yeasts and molds, and filamentous bacteria. All collected specimens were incubated at $37^{\circ} \mathrm{C}$ (for bacteria) or $30^{\circ} \mathrm{C}$ (for fungi) and examined daily for microorganism growth for 1 week and weekly for up to 1 month. Laboratory analyses consisted of culture, microorganism identification, and drug sensitivity tests. Drug sensitivity tests were performed by agar diffusion method using the subsequent antibiotics according to CLSI guidelines: ampicillin, oxacillin, penicillin G, amoxicillin/clavulanic acid, imipenem, gentamicin, rifampicin, ciprofloxacin, levofloxacin, moxifloxacin, trimethoprim/sulfamethoxazole, teicoplanin, vancomycin, clindamycin, erythromycin, nitrofurantoin, linezolid, quinupristin/dalfopristin, tetracycline, cefoxitin, ceftriaxone, 
chloramphenicol, piperacillin/tazobactam, cefotaxime, ceftazidime, cefepime, ertapenem, meropenem, amphotericin, fluconazole, voriconazole, flucytosine.[29]

\section{Statistical Analysis}

Statistical analysis was performed using SPSS software for Windows version 22.0 (SPSS, Inc., Chicago, IL). The 2-tailed unpaired t-test and Pearson Chi-square test performed at the $95 \%$ confidence interval were used to assess differences in continuous and categorical variables. A probability value of $<0.05$ was considered statistically significant. The results are presented as the means \pm standard deviations (SDs) unless otherwise indicated.

\section{Results}

\section{Demographic and ocular characteristics in SJS patients}

General demographics and clinical ocular characteristics of the enrolled patients are shown in Table 1. A total of 30 eyes from 30 patients with SJS were assessed. The average age was $47.8 \pm 16.5(18-71)$ years and the disease onset age was $34.6 \pm 17.8(7-66)$ years. The disease duration was $13.1 \pm 12.1$ ( 0 - 52) years. $9(30.0 \%)$ and $21(70.0 \%)$ patients were male and female, respectively. Cold medications were the most common cause for SJS, followed by antibiotics and other medications, such as antiepileptic drugs. Fourteen (46.7\%) patients had past experience of infectious keratitis. Nearly $70 \%$ of all patients were using topical medications, such as corticosteroids, cyclosporine or antibiotics, at the time when conjunctival swab culture sampling was performed. Schirmer test revealed an average of $6.3 \pm$ $6.3 \mathrm{~mm}$ while the tear break-up time (TBUT) was $3.4 \pm 1.1$ seconds. Matrix metalloproteinase 9 (MMP9) was positive in $24(80.0 \%)$ eyes. The average chronic ocular surface complications score (COCS) was 8.4 \pm 3.3 . Low COCS was considered when the score ranged between 0 and 7 , while a score of 8 or higher was defined as high COCS.[24, 25] 10 (33.3\%) and 20 (66.7\%) eyes were low and high COCS, respectively. 
Table 1

General Demographics and Clinical Characteristics

\begin{tabular}{|c|c|}
\hline Age (years) & $47.8 \pm 16.5(18-71)$ \\
\hline Onset Age (years) & $34.6 \pm 17.8(7-66)$ \\
\hline Disease Durations (years) & $13.1 \pm 12.1(0-52)$ \\
\hline Gender (Male : Female) & $9(30.0 \%): 21(70.0 \%)$ \\
\hline Cause of SJS (Cold drugs : Antibiotics : Others) & $11(36.7 \%): 6(20.0 \%): 13(43.3 \%)$ \\
\hline History of Infectious Keratitis & $14(46.7 \%)$ \\
\hline \multicolumn{2}{|l|}{ Initial Use of Topical Medications } \\
\hline Corticosteroid & $21(70.0 \%)$ \\
\hline Cyclosporine & $20(66.7 \%)$ \\
\hline Antibiotics & $20(66.7 \%)$ \\
\hline Schirmer test (millimeters) & $6.3 \pm 6.3(0-30)$ \\
\hline TBUT (seconds) & $3.4 \pm 1.1(1.4-5.6)$ \\
\hline Positive MMP9 & $24(80.0 \%)$ \\
\hline cocs (score) & $8.4 \pm 3.3(0-14)$ \\
\hline Low $(0-7)$ (patients) & $10(33.3 \%)$ \\
\hline High ( $\geq 8$ ) (patients) & $20(66.7 \%)$ \\
\hline
\end{tabular}

The representative photos of low $(0-7)$ and high $(\geq 8)$ COCS are shown in Figure 1. Figures $1 \mathrm{~A}$ and $1 \mathrm{~B}$ represent low COCS patients and are photos of a female subject's left eye with a COCS score of 3 who had been diagnosed with sulfasalazine-related SJS at the age of 49. Her left eye was observed to have 12 clock hours of corneal neovascularization and nasal corneal opacity in absence of abnormal eyelids, chronic conjunctival hyperemia and symblepharon (Fig. 1A). Under cobalt blue filter examination after fluorescein application, superficial punctate epithelial erosions were observed in the inferior $2 / 3$ of the cornea (Fig. 1B). Figures 1C and 1D represent high COCS and are photos of a female subject's left eye with a COCS score of 10 who had an onset of SJS at age 44 after taking cold medications. Her left eye showed conjunctivalization at the upper $2 / 3$ of the cornea due to partial limbal stem cell deficiency with diffuse corneal haze, chronic conjunctival hyperemia, severe eyelid meibomian gland dysfunctions with trichiasis and lateral symblepharon at both upper and lower portions (Fig. 1C). Under cobalt blue filter 

(Fig. 1D).

\section{Isolation And Drug Resistance Results Of Conjunctival Swab Cultures}

The initial microbial isolation results of conjunctival swab culture samplings are shown in Table 2. 13 (41.9\%) patients had no growth of any microorganisms, while $17(58.1 \%)$ patients were observed to have positive culture results. A total of 27 types of different microorganisms were isolated. Among them, $81.5 \%$ and $11.1 \%$ were gram positive and negative bacteria, respectively, while $7.4 \%$ was fungus origin. Among gram positive bacteria, Coagulase-negative Staphylococci (45.5\%) and Corynebacterium species $(40.9 \%)$ were predominantly observed. Among Coagulase-negative Staphylococci, Staphylococcus epidermidis (60\%) was most commonly isolated followed by Staphylococcus hominis (30\%). Among gram negative bacteria, Klebsiella pneumonia (33.3\%), Stenotrophomonas maltophilia (33.3\%) and Escherichia coli (33.3\%) were observed. All the isolated fungi were Candida. 
Table 2

Initial Microorganism Isolation Results of Conjunctival Swab Culture

\begin{tabular}{|ll|}
\hline Negative Culture (patients) & $\mathbf{1 3}$ (41.9\%) \\
\hline Positive Culture (patients) & $17(58.1 \%)$ \\
\hline Total Number of Isolated Microorganisms & 27 \\
\hline Gram Positive & $22(81.5 \%)$ \\
\hline Coagulase-negative Staphylococci & 10 \\
\hline Staphylococcus epidermidis & 6 \\
\hline Staphylococcus hominis & 3 \\
\hline Staphylococcus haemolyticus & 1 \\
\hline Corynebacterium species & 9 \\
\hline Staphylococcus aureus & 1 \\
\hline Streptococcus viridans & 1 \\
\hline Penicillum species & 1 \\
\hline Gram Negative & $3(11.1 \%)$ \\
\hline Klebsiella pneumoniae & 1 \\
\hline Stenotrophomonas maltophilia & 1 \\
\hline Escherichia coli & 1 \\
\hline Fungus & $2(7.4 \%)$ \\
\hline Candida & 2 \\
\hline
\end{tabular}

The number of patients according to isolated number of microorganisms are shown in Table 3. Positive culture of a single type of microorganism was seen in 12 (70.6\%) of the 17 patients with positive culture results, in which Corynebacterium species was most commonly isolated followed by Staphylococcus epidermidis. Two and three types of microorganism isolations were observed in $2(11.8 \%)$ and 3 (17.6\%) patients, respectively. All fungi were isolated in mixture with other bacteria. 
Table 3

Number Patients According to Number of Isolated Microorganisms

\begin{tabular}{|ll|}
\hline One Isolation (patients) & $\mathbf{1 2}$ (70.6\%) \\
\hline Corynebacterium species & 4 \\
\hline Staphylococcus epidermidis & 3 \\
\hline Staphylococcus hominis & 1 \\
\hline Staphylococcus aureus & 1 \\
\hline Streptococcus viridans & 1 \\
\hline Penicillum species & 1 \\
\hline Klebsiella pneumoniae & 1 \\
\hline Two Isolations (patients) & $2(11.8 \%)$ \\
\hline Corynebacterium species + Coagulase-negative Staphylococci & 2 \\
\hline Three Isolations (patients) & $3(17.6 \%)$ \\
\hline Corynebacterium species + Coagulase-negative Staphylococci & 1 \\
\hline+ Stenotrophomonas maltophilia & \\
\hline Corynebacterium species + Coagulase-negative Staphylococci & 1 \\
\hline+ Candida & 1 \\
\hline Corynebacterium species + Escherichia coli + Candida & 1 \\
\hline
\end{tabular}

Drug resistance was observed in several microorganisms. Among Coagulase-negative Staphylococci, $80 \%$ revealed resistance to penicillin, oxacillin or ampicillin, $70 \%$ against levofloxacin, moxifloxacin or ciprofloxacin, $50 \%$ against erythromycin, $30 \%$ against gentamicin, $20 \%$ against clindamycin and $10 \%$ against tetracycline. Among the isolated Corynebacterium species, $66.7 \%$ revealed resistance to clindamycin, 55.6\% against erythromycin, 33.3\% against penicillin, oxacillin or ampicillin, $22.2 \%$ against chloramphenicol and $11.1 \%$ against gentamicin. However, all isolated Coagulase-negative Staphylococci and Corynebacterium species were susceptible to vancomycin. The solely isolated Staphylococcus aureus was observed to have resistance to ampicillin, penicillin G, gentamicin, ciprofloxacin, levofloxacin, moxifloxacin, clindamycin, and erythromycin, but susceptible to vancomycin and linezolid. The solely isolated Escherichia coli was resistant to trimethoprim/sulfamethoxazole and ciprofloxacin, while susceptible to levofloxacin and moxifloxacin. The isolated Streptococcus viridans, Penicillium species, Klebsiella pneumonia, Stenotrophomonas maltophilia and Candida did not reveal any drug resistance.

\section{Tear MMP9 is associated with positive conjunctival swab culture results}


$13(41.9 \%)$ patients had negative culture results (negative group) while 17 (58.1\%) patients had positive culture results (positive group). The average ages were $44.6 \pm 17.8$ and $50.2 \pm 15.6$ years in negative and positive groups, respectively (unpaired t-test, $P=0.370$ ). SJS onset ages were $31.9 \pm 20.5$ and $36.9 \pm 15.5$ years in negative and positive groups, respectively (unpaired t-test, $P=0.469$ ). The disease durations were $12.6 \pm 14.4$ and $13.6 \pm 10.3$ years in negative and positive groups, respectively (unpaired t-test, $P=$ 0.835). The negative group consisted 4 male and 9 female patients, while the positive group included 5 male and 12 female patients (Pearson Chi-square test, $P=0.936$ ). There was no difference regarding cocs between groups (negative group $8.7 \pm 3.2$ versus (vs.) positive group $8.1 \pm 3.4$, unpaired t-test, $P=$ 0.644 , Fig. 2A). Also, no significant difference in Schirmer test (negative group $5.4 \pm 4.0 \mathrm{~mm}$ vs. positive group $6.9 \pm 7.7 \mathrm{~mm}$ ) and TBUT (negative group $3.8 \pm 1.0 \mathrm{sec}$ vs. positive group $3.1 \pm 1.1 \mathrm{sec}$ ) were observed between groups (Unpaired t-test, $\mathrm{P}=0.511$ and 0.261 , respectively, Fig. 2B and 2C). Positive for tear MMP9 was observed significantly more in the positive group compared to the negative group (100.0\% vs $75.0 \%$, respectively, Pearson Chi-square test, $P=0.040$, Fig. 2D). There was no difference in the use of topical medications, such as corticosteroids, cyclosporine or antibiotics, and in the history of prior infectious keratitis (Pearson Chi-square test, $\mathrm{P}>0.05$, Fig. 2E and 2F).

\section{Topical Cyclosporine Is Associated With Persistent Positive Culture Results}

A total of 13 patients had repetitive conjunctival swab cultures over an interval of at least 3 months since last sampling. Among them, 3 (23.1\%) patients experienced a transition from positive to negative culture results (negative-transition group), while 10 (76.9\%) patients had persistent positive culture results (positive-persistence group). The average ages were $44.3 \pm 11.1$ and $51.3 \pm 16.2$ years in the negativetransition and positive-persistence groups, respectively (unpaired t-test, $P=0.507$ ). Disease onset ages were $39.7 \pm 13.4$ and $38.4 \pm 15.9$ in the negative-transition and positive-persistence groups, respectively (unpaired t-test, $P=0.907$ ). Disease durations were $4.7 \pm 7.2$ and $13.9 \pm 6.7$ years in the negativetransition and positive-persistence groups, respectively (unpaired t-test, $P=0.078$ ). The negativetransition group consisted 1 male and 2 female patients, while the positive-persistence group included 3 male and 7 female patients (Comparison between groups, Pearson Chi-square test, $P=0.913$ ). There was no difference regarding COCS between negative-transition ( $9.0 \pm 4.0)$ and positive-persistence group (10.2 \pm 2.3 ) (unpaired t-test, $P=0.512$, Fig. $3 \mathrm{~A}$ ). There was no significant difference in Schirmer test (negativetransition group $3.0 \pm 2.6 \mathrm{~mm}$ vs. positive-persistence group $3.8 \pm 3.4 \mathrm{~mm}$ ) and TBUT (negative-transition group $4.0 \pm 1.1 \mathrm{sec}$ vs. positive-persistence group $2.8 \pm 1.2 \mathrm{sec}$ ) between groups (unpaired t-test, $\mathrm{P}=$ 0.719 and 0.138 , respectively, Fig. 3B and 3 C). Also, positivity for tear MMP9 did not differ between negative-transition group (66.7\%) and positive-persistence group (90.0\%) (Pearson Chi-square test, $\mathrm{P}=$ 0.070 , Fig. 3D). Notably, patients using topical cyclosporine was observed significantly more in the positive-persistence group (90.0\%) compared to the negative-transition group (33.3\%) (Pearson Chisquare test, $P=0.041$, Fig. $3 E$ ). However, there was no difference concerning other topical medications, such topical corticosteroids or antibiotics, between groups (Pearson Chi-square test, $P>0.05$, Fig. 3E). 
Also, the history of prior infectious keratitis was not different between groups (negative-transition group $33.3 \%$ vs. positive-persistence group $60.0 \%$, Pearson Chi-square test, $P=0.416$, Fig. $3 F$ ).

\section{Discussion}

This study presented (1) high conjunctival swab culture positivity rate in Korean SJS patients with predominant isolation of Coagulase-negative Staphylococci and Corynebacterium species, (2) tear MMP9 positivity to be related to positive culture and (3) the association of topical cyclosporine use with persistence in culture positivity. This study demonstrates high conjunctival swab culture positivity of $58.1 \%$ in Korean SJS patients, in which gram positive bacteria prevailed, and Coagulase-negative Staphylococci and Corynebacterium species were the most commonly isolated microorganisms. Though several microorganisms revealed drug resistance, all isolated bacteria were susceptible to vancomycin and all isolated fungus were susceptible to all antifungal agents. While $66.7 \%$ of patients were using topical cyclosporine, tear MMP9 positivity was significantly observed in patients with positive culture results compared to those with negative results. Also, among the 13 patients who had repetitive conjunctival swab cultures, $76.9 \%$ exhibited persistence in positive culture results which was significantly associated with the use of topical cyclosporine.

Several studies observed that the normal conjunctiva harbors diverse microorganisms, which were identified to be mainly composed of Coagulase-negative Staphylococci, Propionibacterium, Corynebacterium species, Lactobacillus, and Streptococcus, and can be easily altered by factors such as age, diabetes, use of contact lens, or presence of ocular surface diseases.[7-14] To date, several methods, such as conventional swab culture or genetic sequencing methods, have aided the identification of these minute amount of microorganisms.[9] Several studies have reported significant differences in conjunctival microbiota of subjects with ocular surface diseases, especially SJS, compared to those of healthy subjects. $[8,11,14]$ With the conventional swab culture method, SJS was found to have positive culture rate of $59-95 \%$, while a healthy conjunctiva exhibits a relatively low positive culture rate of $10-12.9 \% .[8,9,11]$ Also, Venugopal et al. and Kittipibul et al. observed that SJS patients ' conjunctival swab cultures resulted in more diverse bacterial isolates, including Corynebacterium species, Coagulase-negative Staphyloccoci and Streptococcus, compared to healthy subjects where Coagulasenegative Staphyloccoci were mainly isolated. $[8,11]$ In utilizing next-generation sequencing methods, Kittipibul et al. additionally identified SJS patients' conjunctiva to harbor a higher species diversity index and a significantly different microbiome composition, such as higher proportions of Lactobacillus, Bacteroides, Pseudomonas, Staphylococcus, Streptococcus, Bacillus and Acinetobacter, compared to healthy subjects'.[8] Also, Zilliox et al., using next-generation sequencing methods, observed that the ocular surface microbiome is very different according to the type of ocular surface disease present and found a distinct conjunctival microbiome composition of SJS patients with predomination of Staphyloccocus compared to healthy subjects, but found that the species diversity index was higher in healthy subjects which could have been attributed to the different targeting variable regions used for sequencing.[14] Likewise, this study observed the conjunctival swab cultures of SJS patients to have high positivity of $58.1 \%$ with 12 different types of microorganisms isolated. How the ocular surface 
microbiome and ocular surface immunity interact is yet unknown, but studies have seen the importance of their balance and, therefore, given that SJS patients have generally abnormal ocular surfaces, they are prone to the imbalance that subsequently can induce commensal bacteria to become pathobionts and lead to possible opportunistic infections.[10, 19, 20, 22, 23]

Previous studies reported that the conjunctival swab culture positivity of SJS patients was observed in 59 - 95\%.[8, 11, 18] Venugopal et al. found that the most common isolate in SJS conjunctiva was Coagulasenegative Staphylococci followed by Corynebacterium species and that only $7.6 \%$ was observed to have multiple isolates.[11] Likewise, Kittipibul et al. detected gram positive bacteria as the dominant microorganism, where Corynebacterium species was the most common bacteria, and found that only $10 \%$ resulted with multiple isolations.[8] In another study by Frizon et al., they reported similar results of the gram positive bacteria as the main isolated microorganism in SJS conjunctiva where Coagulasenegative Staphylococci was the most common bacteria followed by Corynebacterium species, but observed multiple isolations in $54 \%$ of SJS patients.[18] Similarly to previous studies, this study also observed positive conjunctival culture of SJS patients in $58.1 \%$, where gram positive bacteria was the predominant isolate. Like other studies, Coagulase-negative Staphylococci and Corynebacterium species were most commonly isolated, and among the isolated Coagulase-negative Staphylococci, Staphylococcus epidermidis was mainly detected followed by Staphylococcus hominis and Staphylococcus haemolyticus. However, in this study, the Streptococcus group was isolated in $5.9 \%$ of SJS patients which was relatively low compared to past studies of $7.7-33.3 \%$. This may be attributed to the relatively older age group included in this study. Cavuoto et al. reported that the Streptococcus group proportion is reduced in elder subjects.[13] Also, this study found that the majority of patients had a single type of bacteria isolated while only $29.4 \%$ of patients had more than one type of microorganism isolated. This number of isolations did not differ according to other ocular components, such as COCS, MMP9 or use of topical corticosteroids or antibiotics.

While Venugopal et al. observed that bacteria isolated from healthy conjunctiva revealed drug sensitivity to most antibacterial agents, they identified all isolated bacteria from SJS conjunctiva to be sensitive to gatifloxacin and moxifloxacin, but resistant against ciprofloxacin in Coagulase-negative Staphylococci, ciprofloxacin, tetracycline, cefazolin, and moxifloxacin in Escherichia coli, and tobramycin and gentamicin in Streptococcus pneumonia.[11] Also, they reported that Streptococcus viridans had the highest percentage of drug resistance.[11] Frizon et al. identified low sensitivity to neomycin and penicillin in the Staphylococcus group, only $33 \%$ sensitivity to chloramphenicol in gram negative bacilli and observed the highest antimicrobial resistance in the Streptococcus group.[18] However, this study found the highest percentage of drug resistance in Coagulase-negative Staphylococci, where more than half were resistant to penicillin, oxacillin, ampicillin, levofloxacin, moxifloxacin or ciprofloxacin, and did not observe any drug resistance in Streptococcus viridans. Also, more than half of the isolated Corynebacterium species exhibited resistance to clindamycin and erythromycin. Aside from Escherichia coli that was resistant to trimethoprim/sulfamethoxazole and ciprofloxacin, gram negative bacteria from this study did not reveal any drug resistance. The difference in the drug susceptibility test results regarding the Streptococcus group compared to previous studies may be because of the low culture rate 
of Streptococcus in this study. Also, the difference in drug resistance of the same kind of microorganism among studies may be attributed to the type of topical antibiotics previously or currently used, in which subjects from Frizon et al.[18] mainly used chloramphenicol eye drops but the patients from this study mainly applied topical levofloxacin or moxifloxacin. Although the drug susceptibility results may differ according to studies, it is still consistently found that the isolated conjunctival microorganisms from SJS patients have relatively higher percentage of drug resistance compared to healthy subjects. This high percentage of drug resistance in SJS patients may be attributed to the chronic use of topical antibiotics and, moreover, the abnormal ocular surface environment leading to the abundance of more virulent pathogens.

To the best of our knowledge, this study is the first to identify the association between tear MMP9 positivity and topical cyclosporine use with conjunctival swab culture positivity and its persistency in positive culture in SJS patients, respectively. Despite recent studies concerning conjunctival microbiota, the role of ocular surface microbiota remains unclear. Moreover, most studies have focused on investigating the compositional or alpha/beta-diversity difference of conjunctival microbiota in subjects with various ocular surface diseases compared to healthy subjects rather than possible relative factors. Though a previous study observed higher culture positivity rate in SJS patients with more severe ocular surface scores,[8] this study did not find any significance between COCS and conjunctival swab culture results. In this study, tear MMP9 positivity was more significant in patients with culture positive results compared to those with culture negative results. Also, patients with culture positive persistence had higher tear MMP9 positivity rate, though it was not significant, and consisted more patients using topical cyclosporine than those who experienced a transition to negative culture results. Despite the chronic use of topical corticosteroid or cyclosporine, the inflammatory nature of SJS may not be fully controlled and may instead promote an environment favoring an abnormal growth of conjunctival microorganisms. Though there was no significance concerning the history of prior infectious keratitis, both patients with positive culture and persistent positive culture results have experienced prior infectious keratitis more than patients with negative culture and transition to negative culture results, respectively. A study by Yoshikawa et al. observed increase in interleukin-8 and Granzyme B in SJS patients with more severe ocular surface scores and found their association with conjunctivalization, neovascularization, opacification or keratinization.[26] Likewise, the results from this study suggests that tear MMP9 positivity may reflect not only the presence of ocular surface inflammation but also the possibility of a more aggressive or persisting abnormal conjunctival microbiota.

There are some limitations to this study. First, it is limited by the relatively small study size. However, given that SJS is a rare disease, a recruitment of 30 patients is not a small number and is similar to previous studies regarding SJS conjunctival microbiota. Also, this study has value in that this is the first to investigate the conjunctival microbiota in Korean SJS patients. Another limitation is that this study observed the conjunctival microbiota using the conventional swab culture method. Though genetic sequencing methods provide more precise and broader information, conventional swab culture still has the advantageous of simplicity and practicality. Lastly, this study is limited in that there is no control group. However, previous studies have already observed that there is distinct difference in conjunctival 
microbiota between SJS and healthy subjects. Still, future studies comparing SJS and healthy subjects' conjunctival microbiota and further exploring their associative factors will be beneficial in elucidating the role of ocular surface microbiota.

\section{Conclusion}

In conclusion, this study demonstrated the high culture positivity rate in SJS patients' conjunctiva. Coagulase-negative Staphylococci and Corynebacterium species were the most commonly isolated microorganisms. Tear MMP9 positivity was associated with positive culture whereas use of topical cyclosporine was related to positive culture-persistence. These findings suggest that tear MMP9 positivity in SJS may indicate the presence of an abnormal ocular surface microbiota. Further investigations regarding the associative factors of conjunctival microbiota in SJS patients are necessary in understanding the relation between conjunctival microbiota and ocular surface immunity.

\section{List Of Abbreviations}

COCS: Chronic ocular surface complications score

MMP9: Matrix metalloproteinase 9

SD: Standard deviations

SJS: Stevens-Johnson syndrome

TBUT: Tear break up time

vs.: Versus

\section{Declarations}

Ethics approval and consent to participate: This retrospective study adhered to the ethical standards of the Declaration of Helsinki and was approved by the Institutional Review Boards of Seoul National University Hospital (IRB No. 2102-014-1193, Seoul, Republic of Korea). The informed consent from patients was waived by the IRB because the study was based on the retrospective review of old charts.

Consent for publication: The informed consent from patients was waived by the IRB because the study was based on the retrospective review of old charts.

Availability of data and materials: All data generated or analyzed during this study are included in this published article.

Competing interests: The authors have no conflicts of interest to disclose.

Funding: This study did not receive any funding. 
Authors' contributions: JM participated in writing, analysis, and interpretation of data. YL participated in drafting, writing, and revising the manuscript. MKK participated in study design, writing, analysis, and interpretation of data. All authors have approved the final submitted version and have agreed to be personally accountable for each contribution.

Acknowledgements: This study was supported by the Cooperative Research Program of Basic Medical Science and Clinical Science from Seoul National University College of Medicine (grant no. 80020190256)

\section{References}

1. Lerch M, Mainetti C, Terziroli Beretta-Piccoli B, Harr T: Current Perspectives on Stevens-Johnson Syndrome and Toxic Epidermal Necrolysis. Clin Rev Allergy Immunol 2018, 54(1):147-176.

2. Kohanim S, Palioura S, Saeed HN, Akpek EK, Amescua G, Basu S, Blomquist PH, Bouchard CS, Dart JK, Gai X et al: Stevens-Johnson Syndrome/Toxic Epidermal Necrolysis-A Comprehensive Review and Guide to Therapy. I. Systemic Disease. Ocul Surf 2016, 14(1):2-19.

3. Yoshikawa Y, Ueta M, Fukuoka H, Inatomi T, Yokota I, Teramukai S, Yokoi N, Kinoshita S, Tajiri K, Ikeda T et al: Long-term Progression of Ocular Surface Disease in Stevens-Johnson Syndrome and Toxic Epidermal Necrolysis. Cornea 2020, 39(6):745-753.

4. Kohanim S, Palioura S, Saeed HN, Akpek EK, Amescua G, Basu S, Blomquist PH, Bouchard CS, Dart JK, Gai X et al: Acute and Chronic Ophthalmic Involvement in Stevens-Johnson Syndrome/Toxic Epidermal Necrolysis - A Comprehensive Review and Guide to Therapy. II. Ophthalmic Disease. Ocul Surf 2016, 14(2):168-188.

5. Hall LN, Shanbhag SS, Rashad R, Chodosh J, Saeed HN: The effects of systemic cyclosporine in acute Stevens-Johnson syndrome/toxic epidermal necrolysis on ocular disease. Ocul Surf 2021, 19:128-132.

6. Shanbhag SS, Rashad R, Chodosh J, Saeed HN: Long-Term Effect of a Treatment Protocol for Acute Ocular Involvement in Stevens-Johnson Syndrome/Toxic Epidermal Necrolysis. Am J Ophthalmol 2019, 208:331-341.

7. Dong Q, Brulc JM, lovieno A, Bates B, Garoutte A, Miller D, Revanna KV, Gao X, Antonopoulos DA, Slepak VZ et al: Diversity of bacteria at healthy human conjunctiva. Invest Ophthalmol Vis Sci 2011, 52(8):5408-5413.

8. Kittipibul T, Puangsricharern V, Chatsuwan T: Comparison of the ocular microbiome between chronic Stevens-Johnson syndrome patients and healthy subjects. Sci Rep 2020, 10(1):4353.

9. Willcox MD: Characterization of the normal microbiota of the ocular surface. Exp Eye Res 2013, 117:99-105.

10. Ueta M, lida T, Sakamoto M, Sotozono C, Takahashi J, Kojima K, Okada K, Chen X, Kinoshita S, Honda T: Polyclonality of Staphylococcus epidermidis residing on the healthy ocular surface. J Med Microbiol 2007, 56(Pt 1):77-82. 
11. Venugopal R, Satpathy G, Sangwan S, Kapil A, Aron N, Agarwal T, Pushker N, Sharma N: Conjunctival Microbial Flora in Ocular Stevens-Johnson Syndrome Sequelae Patients at a Tertiary Eye Care Center. Cornea 2016, 35(8):1117-1121.

12. Ozkan J, Nielsen S, Diez-Vives C, Coroneo M, Thomas T, Willcox M: Temporal Stability and Composition of the Ocular Surface Microbiome. Sci Rep 2017, 7(1):9880.

13. Cavuoto KM, Mendez R, Miller D, Galor A, Banerjee S: Effect of clinical parameters on the ocular surface microbiome in children and adults. Clin Ophthalmol 2018, 12:1189-1197.

14. Zilliox MJ, Gange WS, Kuffel G, Mores CR, Joyce C, de Bustros P, Bouchard CS: Assessing the ocular surface microbiome in severe ocular surface diseases. Ocul Surf 2020, 18(4):706-712.

15. Suto $\mathrm{C}$, Morinaga $\mathrm{M}$, Yagi T, Tsuji C, Toshida H: Conjunctival sac bacterial flora isolated prior to cataract surgery. Infect Drug Resist 2012, 5:37-41.

16. Hori Y, Maeda N, Sakamoto M, Koh S, Inoue T, Tano Y: Bacteriologic profile of the conjunctiva in the patients with dry eye. American journal of ophthalmology 2008, 146(5):729-734.

17. Lee SH, Oh DH, Jung JY, Kim JC, Jeon CO: Comparative ocular microbial communities in humans with and without blepharitis. Invest Ophthalmol Vis Sci 2012, 53(9):5585-5593.

18. Frizon L, Araujo MC, Andrade L, Yu MC, Wakamatsu TH, Hofling-Lima AL, Gomes JA: Evaluation of conjunctival bacterial flora in patients with Stevens-Johnson Syndrome. Clinics 2014, 69(3):168172.

19. Ueta M, Kinoshita S: Ocular surface inflammation is regulated by innate immunity. Prog Retin Eye Res 2012, 31(6):551-575.

20. Sotozono C, Inagaki K, Fujita A, Koizumi N, Sano Y, Inatomi T, Kinoshita S: Methicillin-resistant Staphylococcus aureus and methicillin-resistant Staphylococcus epidermidis infections in the cornea. Cornea 2002, 21(7 Suppl):S94-101.

21. Nouri M, Terada $\mathrm{H}$, Alfonso EC, Foster CS, Durand ML, Dohlman CH: Endophthalmitis after keratoprosthesis: incidence, bacterial causes, and risk factors. Arch Ophthalmol 2001, 119(4):484489.

22. Sharma N, Venugopal R, Singhal D, Maharana PK, Sangwan S, Satpathy G: Microbial Keratitis in Stevens-Johnson Syndrome: A Prospective Study. Cornea 2019, 38(8):938-942.

23. Bagga B, Motukupally SR, Mohamed A: Microbial keratitis in Stevens-Johnson syndrome: Clinical and microbiological profile. Ocul Surf 2018, 16(4):454-457.

24. Sotozono C, Ang LP, Koizumi N, Higashihara H, Ueta M, Inatomi T, Yokoi N, Kaido M, Dogru M, Shimazaki $\mathrm{J}$ et al: New grading system for the evaluation of chronic ocular manifestations in patients with Stevens-Johnson syndrome. Ophthalmology 2007, 114(7):1294-1302.

25. Kim DH, Yoon KC, Seo KY, Lee HS, Yoon SC, Sotozono C, Ueta M, Kim MK: The role of systemic immunomodulatory treatment and prognostic factors on chronic ocular complications in StevensJohnson syndrome. Ophthalmology 2015, 122(2):254-264. 
26. Yoshikawa Y, Ueta M, Nishigaki H, Kinoshita S, Ikeda T, Sotozono C: Predictive biomarkers for the progression of ocular complications in chronic Stevens-Johnson syndrome and toxic Eeidermal necrolysis. Sci Rep 2020, 10(1):18922.

27. Jun JH, Lee $\mathrm{YH}$, Son MJ, Kim H: Importance of tear volume for positivity of tear matrix metalloproteinase-9 immunoassay. PLoS One 2020, 15(7):e0235408.

28. Sambursky R, Davitt WF, 3rd, Friedberg M, Tauber S: Prospective, multicenter, clinical evaluation of point-of-care matrix metalloproteinase-9 test for confirming dry eye disease. Cornea 2014, 33(8):812-818.

29. Bauer AW, Kirby WM, Sherris JC, Turck M: Antibiotic susceptibility testing by a standardized single disk method. Am J Clin Pathol 1966, 45(4):493-496.

\section{Figures}

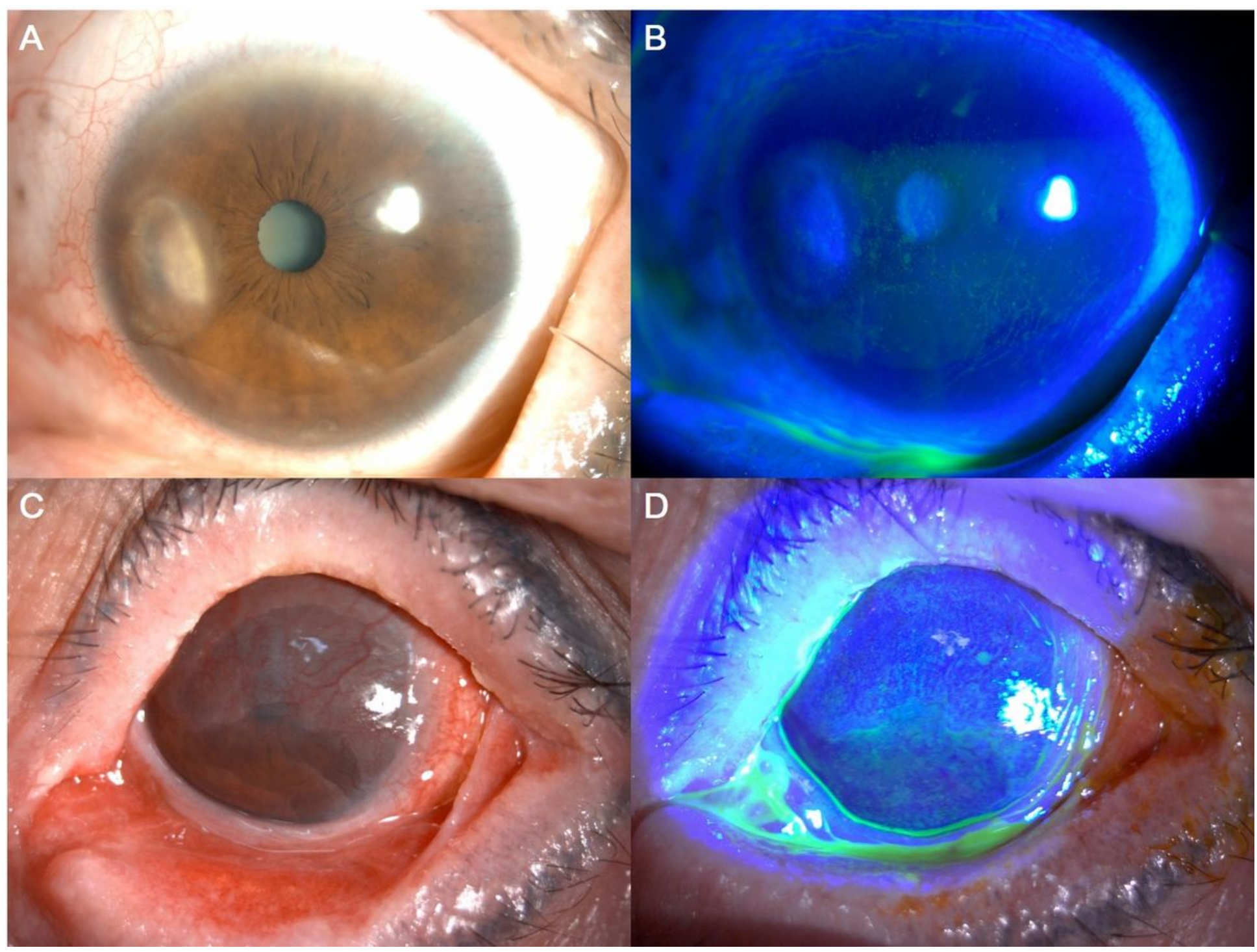

Figure 1 
The representative photos of low $(0-7)$ and high $(\geq 8)$ COCS. Figures A and B represents low $(0-7)$ COCS and are photos of a female subject's left eye with a COCS score of 3 who had been diagnosed with sulfasalazine-related SJS at the age of 49 . Her left eye exhibited 12 clock hours of corneal neovascularization and nasal corneal opacity but without severe meibomian gland dysfunctions, chronic conjunctival hyperemia nor symblepharon (A). Under cobalt blue filter examination after fluorescein application, superficial punctate epithelial erosions were observed in the inferior $2 / 3$ of the cornea (B). Figures $C$ and $D$ represents high $(\geq 8)$ COCS and are photos of a female subject's left eye with a COCS score of 10 who had an onset of SJS at age 44 after taking cold medications. Her left eye had conjunctivalization at the upper $2 / 3$ of the cornea due to limbal stem cell deficiency with diffuse corneal haze, chronic conjunctival hyperemia, severe eyelid meibomian gland dysfunctions with trichiasis and lateral symblepharon at both upper and lower portions (C). Under cobalt blue filter examination after fluorescein application, diffuse superficial punctate epithelial erosions were observed (D). COCS: Chronic Ocular Surface Complications Score, SJS: Stevens-Johnson syndrome
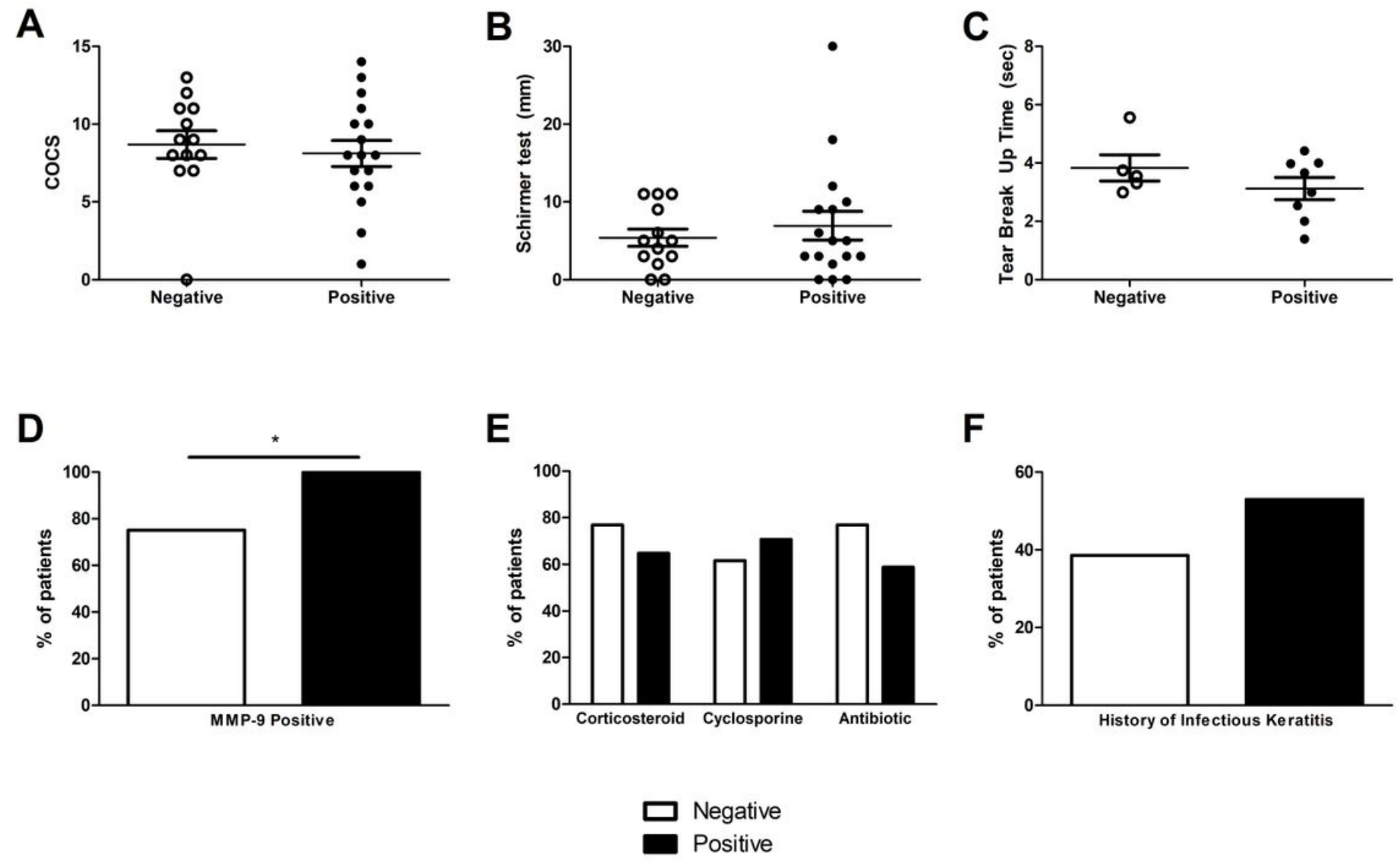

$\mathbf{F}$

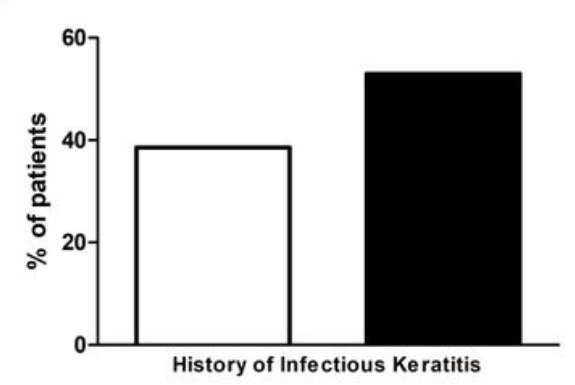

\section{Figure 2}

Clinical characteristic differences between patients with negative and positive culture. 13 (41.9\%) patients had negative culture results (negative group) while 17 (58.1\%) patients had positive culture results (positive group). The COCS was $8.7 \pm 3.2$ and $8.1 \pm 3.4$ in patients with negative and positive culture results, respectively (unpaired t-test, $P=0.644)(A)$. No significant difference was observed in tear secretion by Schirmer test between groups (negative group $5.4 \pm 4.0 \mathrm{~mm}$ vs. positive group $6.9 \pm 7.7 \mathrm{~mm}$, 
unpaired $t$-test, $P=0.511)(B)$. Also, TBUT was not significantly different and were $3.8 \pm 1.0$ and $3.1 \pm 1.1$ seconds in the negative and positive groups, respectively (unpaired t-test, $P=0.261$ ) (C). Tear MMP9 positivity was higher in patients with positive culture (100\%) compare to those with negative culture $(75 \%)$ results (Pearson Chi-square test, $P=0.040)(D)$. There was no difference in the use of topical medications, such as corticosteroids, cyclosporine or antibiotics $(\mathrm{E})$, and in the history of prior infectious keratitis (F) (Pearson Chi-square test, $\mathrm{P}>0.05$ ). COCS: Chronic Ocular Surface Complications Score, TBUT: Tear break up time, MMP9: Matrix metalloproteinase 9, vs: Versus * $P<0.05$, Pearson Chi-square test

A

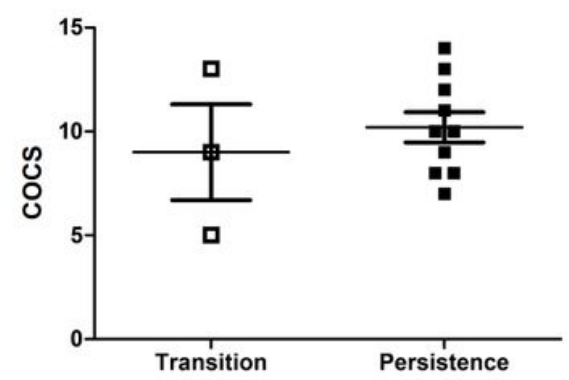

D

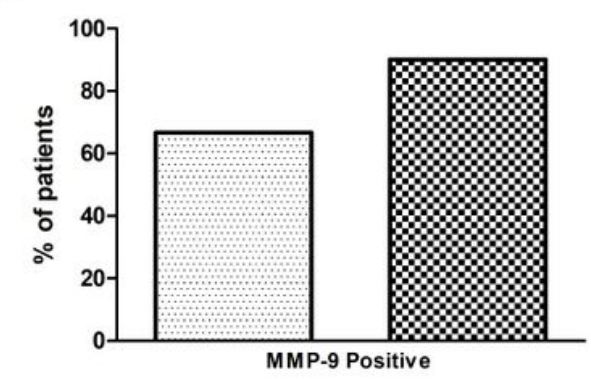

B

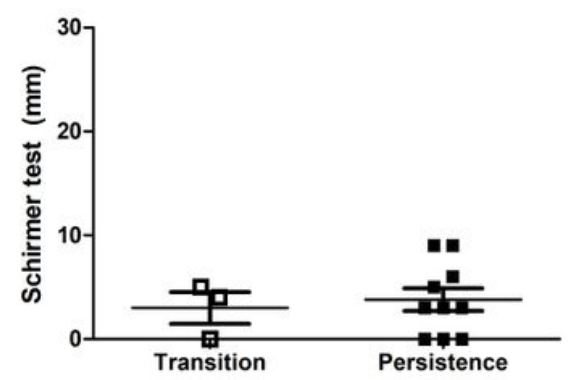

E

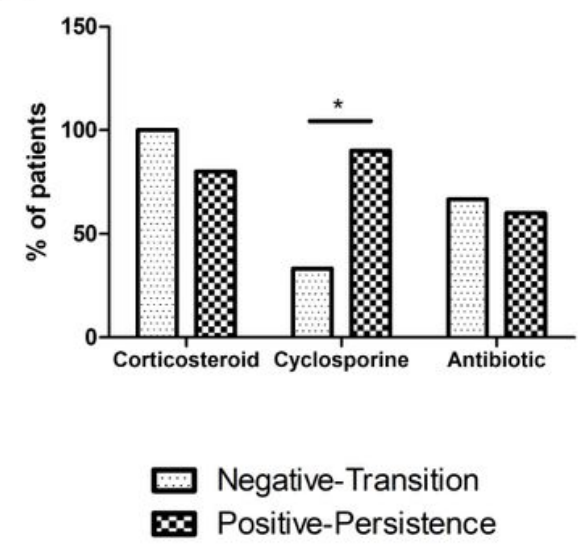

C

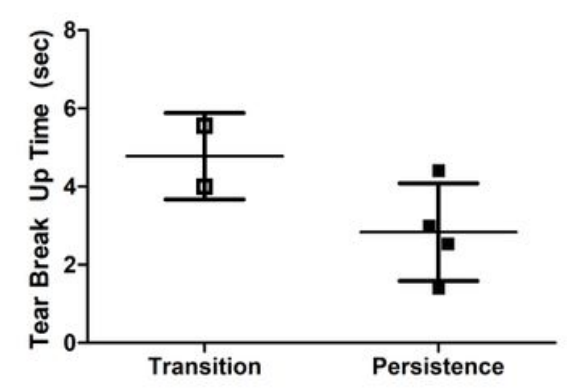

$\mathbf{F}$

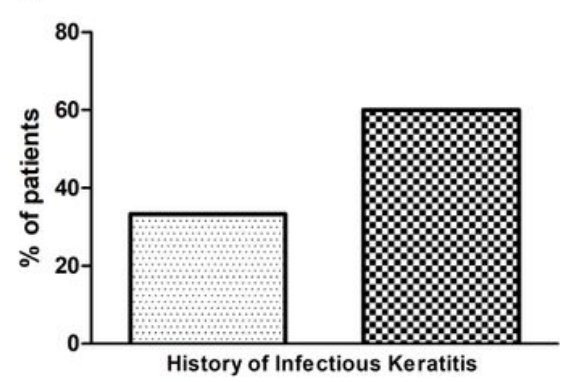

\section{Figure 3}

Clinical characteristic differences between patients with repetitive cultures resulting in negative transition and positive persistence. Among the 13 patients who had repetitive conjunctival swab cultures, 3 (23.1\%) patients experienced a transition from positive to negative culture results (negative-transition group), while 10 (76.9\%) patients had persistent positive culture results (positive-persistence group). There was no difference regarding COCS between groups (negative-transition group $9.0 \pm 4.0$ vs. positive-persistence group $10.2 \pm 2.3$, unpaired t-test, $P=0.512)(A)$. No significant difference in Schirmer test was observed between groups (negative-transition group $3.0 \pm 2.6 \mathrm{~mm}$ vs. positive-persistence group $3.8 \pm 3.4 \mathrm{~mm}$, unpaired t-test, $P=0.719)(B)$. Also, TBUT did not differ between groups (negative-transition group $4.0 \pm$ $1.1 \mathrm{sec}$ vs. positive-persistence group $2.8 \pm 1.2 \mathrm{sec}$, unpaired t-test, $P=0.138$ ) (C). Positivity for tear MMP9 was not different between negative-transition group (66.7\%) and positive-persistence group (90.0\%) (Pearson Chi-square test, $P=0.070)(D)$. Patients using topical cyclosporine was observed 
significantly more in the positive-persistence group compared to the negative-transition group $(90.0 \%$ vs 33.3\%, respectively, Pearson Chi-square test, $P=0.041$ ), while topical use of corticosteroids and antibiotics did not exhibit any significance (Pearson Chi-square test, $P>0.05)(E)$. History of prior infectious keratitis was not different between groups (negative-transition group $33.3 \%$ vs. positivepersistence group 60.0\%, Pearson Chi-square test, $P=0.416)(F)$. COCS: Chronic Ocular Surface Complications Score, TBUT: Tear break up time, MMP9: Matrix metalloproteinase 9, vs: Versus * $P<0.05$, Pearson Chi-square test 